\title{
ESTUDIO DE LA TECNOLOGÍA DE TVWS EN COLOMBIA Y COMPARACIÓN CON EL MUNDO
}

\section{STUDY OF TVWS TECHNOLOGY IN COLOMBIA AND COMPARISON WITH THE WORLD}

\section{CT. Duban Ferney Aragón Rojas*, CT. Luis Eduardo Gómez García* CT. Gustavo Mauricio Rivera Ortiz*, MSc. William Alexander Cuevas Carrero*}

\author{
* Escuela de Comunicaciones Militares, Departamento de Educación Superior \\ Complementaria, Ingeniería Electrónica y Comunicaciones. \\ Calle 5 con carrera 15 dos caminos, Facatativá, Cundinamarca, Colombia. \\ Teléfono: 57-1 2216336 - 2220950 - 4261499 \\ E-mail: \{dubanaragon910101@hotmail.com, luisgom1990@gmail.com, \\ gustavomauricio18@hotmail.com,william.cuevas@escom.edu.co\}.
}

\begin{abstract}
Resumen: Este artículo trata acerca, del estudio de la tecnología de espacios en blanco (TVWS) en beneficio del Ejército Nacional; como una opción para brindar internet libre en zonas alejadas, entre las frecuencias en la banda de $480 \mathrm{MHz}$ a $698 \mathrm{MHz}$, dadas por la normatividad en Colombia. La orientación que se presentó, es la revisión del estado del arte de la tecnología TVWS en Colombia, frente a los diferentes países en el mundo que han trabajado en esta área; con el fin de llevar a cabo un análisis en cuanto a: regulación, aspectos técnicos, desarrollo de pruebas piloto e implementaciones.
\end{abstract}

Palabras clave: Comparación, Internet, Regulación, Rural, TVWS.

\begin{abstract}
This article deals with the study of white space technology (TVWS) for the benefit of the National Army; as an option to provide free internet in remote areas, between the frequencies in the band $480 \mathrm{MHz}$ to $698 \mathrm{MHz}$, given by regulations in Colombia. The orientation presented is the review of the state of the art of TVWS technology in Colombia, in front of the different countries in the world that have worked in this area; in order to carry out an analysis in terms of: regulation, technical aspects, development of pilot tests and implementations.
\end{abstract}

Keywords: Comparison, Internet, Regulation, Rural, TVWS.

\section{INTRODUCCIÓN}

La función principal del Ejército Nacional, es ejercer soberanía en el territorio nacional, así como la protección de la población civil y sus bienes.
Para cumplir distintas tareas, la Fuerza hace presencia en zonas tanto urbanas como rurales, haciendo movimientos pedestres o motorizados a lugares del país que, por sus condiciones geográficas, son de difícil acceso y por ende en 
algunos de ellos hacen falta los servicios públicos básicos, para las personas que viven en mencionados lugares.

Por esto, se contempla la necesidad del servicio de internet, para las unidades operativas, que es indispensable en la actualidad, ya que permite estar en contacto con el mundo haciendo las labores militares y civiles más sencillas. Sin embargo, en el territorio nacional se tienen asignados canales de frecuencia televisiva a nivel municipal $y$ departamental; donde existen canales de televisión sin utilizar que corresponden a un recurso electrónico que se malgasta.

En consecuencia, se contempla la tecnología TVWS que se describe así:

"Como resultado de la liberación de muchos canales analógicos, debido a la transición de televisión análoga a digital, han quedado libres espacios en el espectro; para que sean usados por dispositivos de baja potencia, que no causan interferencia. Estos espacios se denominan TV White Spaces (TVWS), ya que se encuentran en la banda de frecuencias de televisión". (Delicado, 2016)

Esta tecnología ha sido vista con satisfacción alrededor del mundo, debido a que el uso del espectro que se subutiliza, puede ser útil para el envío de datos a un bajo costo, aspecto que se podría hacer por medio de internet satelital, pero su alto precio hace que sea más difícil para algunos países. Así pues, se expresa que: "TVWS es una alternativa tecnológica que presenta un medio, para resolver el acceso a las Tics de las poblaciones rurales". (Cuevas-Riz, 2017)

A nivel mundial, se han realizado investigaciones acerca de TVWS, específicamente en temas tales como: internet gratuito para los ciudadanos, manejo de software de evaluación de espacios en blanco, reducción en la complejidad, espacios en blanco dinámicos y en tiempo real, planes de implementación, simulación, entre otros aspectos de interés, en los que se ha avanzado en la actualidad.

Del mismo modo, existen pruebas piloto en Colombia realizadas por la ANE (Autoridad Nacional del Espectro) ejecutadas en diversos municipios tales como:

"Dibulla en el departamento de la Guajira, Pamplonita en Norte de Santander y Aguadas
Caldas, específicamente en escuelas rurales para mostrar el desempeño de la tecnología TVWS y su utilidad en el apoyo a la educación". (Suarez, 2018)

Además, existe un proyecto de la ANE titulado Transformación Tecnológica que consiste en:

"Utilizar la tecnología TVWS en el municipio de Mesetas Meta, con el fin de conectar cinco fincas cafeteras y dos escuelas rurales del municipio en busca de la transformación de la región mediante la conexión a internet” (ANE, 2018).

Asimismo, se han llevado a cabo otras investigaciones debido al interés de diversas instituciones en la tecnología TVWS.

De allí que el estudio para la implementación de estas tecnologías en el Ejército Nacional, representa una alternativa para llevar internet a las áreas rurales donde hay tropas en operaciones, con un sin número de fines tanto institucionales como personales. Por consiguiente, se determina como pregunta de investigación: ¿Qué debe incluir un estudio de comparación de la tecnología de espacios en blanco de televisión (TVWS) entre Colombia y el mundo, para determinar si es viable usarla en las unidades del Ejército Nacional localizadas en áreas rurales?, que se toma como punto de partida para plantear soluciones al problema de investigación.

\section{MÉTODOS Y MATERIALES}

\subsection{Enfoque, alcance $y$ diseño de la investigación}

\subsubsection{Enfoque}

El enfoque del artículo, fue cualitativo ya que se caracterizaba por:

"Usar la teoría como marco de referencia, generar teoría a partir de datos empíricos obtenidos y analizados y, por último, utilizar la revisión de la literatura como un aspecto relevante en el desarrollo de la investigación" (Henderson, 2014)

De esta manera, a partir del enfoque cualitativo fue posible, construir información, mediante la recopilación documental de un tema en específico. 


\subsubsection{Alcance}

"Los alcances de la investigación, están dados por la revisión de la literatura y de la perspectiva del estudio. Esta elección, va de acuerdo a los objetivos de los investigadores para combinar los elementos de estudio”. (Hernández, 2014)

Por tanto, el proyecto desarrollado se ajustó al alcance correlacional; ya que consideró:

"Medir el grado de relación entre dos o más variables y posee características tales como: asociar conceptos, permitir predicciones y medir relaciones entre variables.” (Hernández, 2014)

Lo que se logró a partir del artículo fue: realizar un estudio de la tecnología TVWS, y considerar su uso en el Ejército Nacional, para que la Fuerza cuente con internet gratuito en zonas rurales que carecen de este servicio en beneficio de las tropas. A través del estado del arte, se determinó la normatividad, aspectos técnicos, pruebas piloto e implementaciones, realizadas en Colombia y en diversas partes del mundo.

\subsubsection{Diseño de la investigación}

El diseño de la investigación trata de:

"El plan, la estructura y las estrategias que se usan para obtener respuestas a la pregunta de investigación. Los diseños implican partir de la teoría, señalar como se obtendrán los datos, cuantos registros u observaciones se realizarán y como se analizará la información obtenida".

El diseño de este artículo, es de tipo investigaciónacción debido a:

"Que busca resolver problemas cotidianos e inmediatos y mejorar prácticas concretas, se centra principalmente en aportar información que guie la toma de decisiones, programas, procesos o reformas estructurales" (Reidl, 2014).

Este tipo de diseño fue contemplado, debido a su relación, con el estudio que se llevó a cabo sobre tecnología TVWS y su implicación en el bienestar de las tropas del Ejército Nacional.

\subsubsection{Participantes}

Los participantes, que hicieron parte de esta investigación fueron: Los integrantes del grupo de estudio, el director del proyecto, los asesores del proyecto, las personas entrevistadas en el marco del proyecto y las tropas del Ejército Nacional, que se beneficiaran con el proyecto.

\subsubsection{Instrumentos empleados}

Los instrumentos empleados:

"Resumen la labor previa de una investigación. Los principales instrumentos que se utilizan en la recopilación de datos, sin importar la modalidad investigativa son los siguientes: observación, recopilación o investigación documental, entrevista, cuestionario o encuesta". (Cerda, 1991)

Las herramientas, usadas en este artículo principalmente son: la recopilación documental, a través de la indagación del estado del arte en Colombia y en diferentes países del mundo, sobre la tecnología TVWS, y las entrevistas realizadas a personas que hacen parte de la Escuela de Comunicaciones Militares de Facatativá, acerca del concepto de espacios en blanco de TV, su posible aplicación en el Ejército Nacional y recomendaciones.

\subsection{Recolección de datos}

"En las investigaciones cualitativas, se requiere de la lectura, interpretación y apropiación de la información relacionada con el tema objeto de estudio. Es por esto, que se contemplan fuentes documentales en formato digital, por ejemplo, libros digitales, revistas electrónicas, ponencias, informes de investigaciones, entre otros" (Orellana \& Sanchez, 2006)

Debido a esto, fue examinada la información disponible de la tecnología TVWS de diferentes fuentes confiables, llevando a cabo una revisión de la situación actual del tema de estudio. Las entrevistas abiertas, constituyeron una fuente de información flexible, que dio pautas en el desarrollo del artículo.

En las entrevistas realizadas se manifestó, que los espacios en blanco de TV, representan un área del conocimiento ampliamente usada en la actualidad 
Revista Colombiana de Tecnologías de Avanzada

en proyectos de orden nacional, ha sido estudiada por diferentes universidades con el fin de desarrollar proyectos para el aprovechamiento del espectro disponible, y para el caso del Ejército Nacional podría beneficiar a las tropas en las áreas de comunicación, comando y control.

\section{PROCEDIMIENTO}

\subsection{Regulación}

En primer lugar, se contemplaron 14 países que usaban los espacios en blanco de TV incluido Colombia y hacen parte de los cinco continentes. De esta manera, se indican los organismos reguladores y las frecuencias disponibles de espacios en blanco en los países seleccionados. Estos valores se encuentran consignados en la regulación de cada país, debido a que se ha difundido esta tecnología a lo largo del mundo; y se ha creado la normatividad de la tecnología TVWS.

De esta manera, puede evidenciarse las diferencias significativas que existieron entre la asignación de frecuencias, que se usan en la tecnología TVWS en diferentes lugares del mundo, en las que se pueden llevar a cabo diferentes aplicaciones según sea la necesidad.

\subsection{Aspectos técnicos}

"En la regulación TVWS, se contemplaba la protección del contorno, posicionamiento, limites fuera de banda (OOB), ciclo de actualización y otros parámetros importantes, ya que para implementar TVWS, en países específicos los parámetros difieren para diversos territorios. (Oh et al., 2016)

Los aspectos técnicos en algunos países, que se encontraban en la regulación se especifican posteriormente

\subsection{Proyectos Piloto e implementaciones}

Alrededor del mundo, se han desarrollado diversas pruebas piloto e implementaciones, con el fin de innovar en el área de la tecnología TVWS. A continuación, se mencionan investigaciones llevadas a cabo recientemente en diversas partes del mundo. En Alemania:

"Se ha explorado la tecnología TVWS para el uso de redes celulares utilizando la metodología europea CEPT ECC SE 43, con el fin de determinar el rendimiento de este tipo de redes, utilizando simulación de múltiples celdas y usuarios para evaluar la interferencia". (Dudda et al., 2012)

En cuanto, a los avances realizados en Australia:

"Se habla sobre el software de código abierto WEST, que permitió determinar la cantidad de espacios en blanco en una zona establecida y tuvo pruebas de funcionamiento en Canadá y Australia. La principal ventaja de WEST, fue la posibilidad de ajustar las reglas existentes e hipotéticas, además del diseño modular y de alto nivel de WEST, que sirve para facilitar la programación por parte del usuario" (Harrison et al., 2015)

Asimismo, en un estudio realizado en Canadá se especifica:

"El uso de la radio cognitiva y los espacios en blanco de televisión, como solución viable para que los usuarios secundarios puedan emplear la tecnología TVWS. Para esto, se diseñó, un prototipo de banco de pruebas en tiempo real de la transmisión de usuarios secundarios en TVWS. De esta forma, se utiliza un dispositivo USRP y el software GNU Radio, el receptor de radio definida por software (SDR) y un analizador de espectros".(Hassan \& Ariful, 2019)

Por otro lado, en Colombia a través de una investigación realizada, se expresa que:

"En el país existen bases jurídicas y regulatorias para el uso de bandas entre 470 a $698 \mathrm{MHz}$ que permite la implementación de la tecnología IOT (Internet de las cosas), sin embargo, existen desafíos frente a su gestión y regulación, en cuanto a mecanismos de control de dispositivos de TVWS. Para esto, se desarrolla una red maestro-esclavo con radios configurables SDR y utilizando el software Python". (Roncancio, 2017)

Por otra parte, en:

"Estados Unidos se expone en un estudio acerca de White-Fi, que se refiere a Wifi desplegado en espacios en blanco de TV. Este nuevo tipo de tecnología debe cumplir con requerimientos de protección en la recepción de TV. De esta manera, la optimización enfrentaba 
desafíos en la disponibilidad de canales, y la calidad de los enlaces. Para esto se propuso un algoritmo para optimizar el rendimiento de la red White.Fi. Se utilizaban como referencia las redes de las ciudades de Denver y Columbus en los EE UU'. (Gopal et al., 2018)

Al mismo tiempo:

"Finlandia planteaba un método para configurar el nivel de potencia de transmisión de una red celular que operaba en TVWS, que según FCC no debe superar los $4 \mathrm{~W}$, sin afectar los receptores de TV”. (Cho et al., 2012)

Por el contrario, en Japón:

"Se ha utilizado la tecnología TVWS en vehículos ya que los fabricantes de automóviles se encontraban interesados, en sistemas de comunicación inalámbricos de vehículo a vehículo, a través de espacios en blanco de TV, este sistema poseía una base de datos de geolocalización, detección de espectro y se efectuaban pruebas de campo respecto al rendimiento de este tipo de sistema". (Altintas et al., 2016)

Igualmente, en Kenia una prueba de banda ancha:

"Con espacios en blanco en Nanyuki ha demostrado ser exitosa como alternativa, para proporcionar internet en áreas remotas y en lugares difíciles". (CA, 2014)

También, en Malawi se analizó:

"El rendimiento de los espacios en blanco de TV en la banda UHF. La comparación que se realizó con otros servicios de banda ancha fija, dando como resultado que los servicios TVWS demostraron ser 2,6 veces mejores respecto, a las tasas de datos dadas en operación- La red operativa más larga es de 18,56 Km con SNR de $10 \mathrm{~dB}$. Sin embargo, el rango funcional probado en este momento fue de $7.5 \mathrm{~km}$ (AirWing), con una SNR de 24.7, que representó resultados favorables". (Mikeka et al., 2014)

Sin embargo, en Nueva Zelanda:

"Se usó la radio cognitiva como solución, para satisfacer la demanda de rendimiento de las redes ADHOC vehiculares denominadas VANET. Fue posible ofrecer aplicaciones, de
Revista Colombiana de Tecnologías de Avanzada

información y entretenimiento en espacios en blanco de TV con radio cognitiva, para mantener las conexiones entre proveedores y usuarios de servicios de aplicación. Se utilizaron múltiples canales en el espectro, para evitar las pérdidas de paquetes, probando su funcionamiento mediante el software OMNET++, revelando una mejora de un $91 \%$ en la recepción de paquetes". (Abeywardana et al., 2018)

Además, en el Reino Unido, existió un estudio sobre:

"Una prueba piloto que presentó buenos resultados, en redes de área local inalámbricos interiores. Aunque existió una gran disponibilidad de tecnología TVWS en Londres, se logró ver afectada el área localizad fuera de Londres debido a la variabilidad" (Holland et al., 2015)

En Singapur, se presentó un prototipo, para una prueba piloto de espacios en blanco de TV:

"El dispositivo contaba con un diseño de hardware y software, con capacidad de acceso a base de datos, con funciones de radio cognitiva y cumplía con las condiciones de emisiones fuera de banda". (Song et al., 2012)

Asimismo, en Sudáfrica se llevaron a cabo mediciones de campo:

"Para evaluar aspectos claves en la operación de las redes de espacios en blanco y la posible interferencia con la recepción de canales de TV, de esta forma se analizaron los resultados de campo, así se concluyó que puede existir la posibilidad de interferencia en un rango de $200 \mathrm{~m}$, que representó un resultado positivo". (Masonta \& Mfupe, 2013)

Así pues, en Vietnam se realizó una prueba TVWS:

"Que consistió en una red de 5 ciudades, en zonas tanto rurales como urbanas. Con Wifi se obtuvo una cobertura rural de $7 \mathrm{~km}$, en la montaña de 4km, en la región urbana de 3-6,5 km y en los suburbios de 7,5 Km. Al aumentar la altura de la antena y usar la tecnología TVWS el radio de cobertura aumentó a $20 \mathrm{~km}$. Por último, este sistema poseía una base de datos de espacios en blanco avalada por la FCC". (Huu, 2015) 


\section{RESULTADOS}

\subsection{Resultados de la regulación}

En la Tabla 1, se observan los países elegidos para el estudio, los cuales fueron: Alemania, Australia, Canadá, Colombia, Estados Unidos, Finlandia, Japón, Kenia, Malawi, Nueva Zelanda, Reino Unido, Singapur, Sudáfrica y Vietnam. Estos países fueron seleccionados, debido a su representativa participación en el desarrollo de la tecnología TVWS, sin embargo, algunos de ellos se encontraban desarrollando un borrador de la regulación tales como Malawi y Kenia.

No obstante, algunos países como Estados Unidos, Canadá, Reino Unido, Japón, Singapur, Sudáfrica o Colombia presentaban una regulación fortalecida en cuanto a la tecnología de espacios en blanco de TV. También, se encuentran en la Tabla 1 las frecuencias disponibles para espacios en blanco de TV en estas naciones, que varían considerablemente entre los diferentes países.

Es significativo destacar, que algunos de los países determinaban sus frecuencias en relación a las entidades reguladoras más importantes tales como Ofcom y FCC, mientras otros elegían las frecuencias de acuerdo a los servicios que se prestaban específicamente y requerían de una protección especial como por ejemplo, la sub- banda de radio astronomía para el caso de Sudáfrica, que implicaba una restricción en el ancho de banda que se podía usar en aplicaciones de espacios en blanco de TV, cuyo fin fundamental es evitar la interferencia con los usuarios primarios.

En adición, Colombia se destacaba por haber sido el primer país en culminar la regulación de TVWS en Latinoamérica, para esto seleccionó una frecuencia disponible para espacios en blanco, guiándose por la normatividad dada por la FCC de Estados Unidos. Por último, es importante resaltar la importancia del ITU a nivel mundial, como entidad reguladora de las pautas sobre el uso del espectro.
Tabla 1: Entidades reguladoras y frecuencias

\begin{tabular}{|c|c|c|}
\hline País & $\begin{array}{c}\text { Entidad } \\
\text { Reguladora }\end{array}$ & $\begin{array}{l}\text { Frecuencias disponibles } \\
\text { para espacios en blanco }\end{array}$ \\
\hline Alemania & BNetzA & $470-790 \mathrm{MHz}$ \\
\hline Australia & ACMA & $470-698 \mathrm{MHz}$ \\
\hline Canada & Industry Canada & $\begin{array}{l}54-60 \mathrm{MHz} \\
76-88 \mathrm{MHz} \\
470-608 \mathrm{MHz} \\
614-698 \mathrm{MHz}\end{array}$ \\
\hline Colombia & ANE & $400-698 \mathrm{MHz}$ \\
\hline Estados Unidos & FCC & $\begin{array}{l}54-72 \mathrm{MHz} \\
76-88 \mathrm{MHz} \\
174-216 \mathrm{MHz} \\
470-698 \mathrm{MHz}\end{array}$ \\
\hline Finlandia & FICORA & $470-790 \mathrm{MHz}$ \\
\hline Japón & NICT & $470-710 \mathrm{MHz}$ \\
\hline Kenia & $\mathrm{CA}$ & $470-694 \mathrm{MHz}$ \\
\hline Malawi & MACRA & $470-694 \mathrm{MHz}$ \\
\hline Nueva Zelanda & RSM & $470-790 \mathrm{MHz}$ \\
\hline Reino Unido & Ofcom & $470-790 \mathrm{MHz}$ \\
\hline Singapur & iDA & $\begin{array}{l}181-188 \mathrm{MHz} \\
209-223 \mathrm{MHz} \\
614-622 \mathrm{MHz} \\
630-710 \mathrm{MHz} \\
718-742 \mathrm{MHz} \\
750-774 \mathrm{MHz} \\
790-806 \mathrm{MHz}\end{array}$ \\
\hline Sudáfrica & ICASA & $\begin{array}{c}\text { 470-694 MHz } \\
\text { Excluyendo la subbanda } \\
606-614 \mathrm{MHz}\end{array}$ \\
\hline Vietnam & ARFM & $470-790 \mathrm{MHz}$ \\
\hline
\end{tabular}

Nota. Datos tomados de las siguientes referencias: (ACMA, 2016; ANE, 2017; CEPT, 2015; Dudda et al., 2012; FCC, 2015; Haji, 2014; Huu, 2015; ICASA, 2018; iDA, 2015; Industry Canada, 2015; Mikeka, 2018; NICT, 2015; Ofcom, 2015; RSM, 2018)

\subsection{Resultados de los aspectos técnicos}

En la regulación de los diferentes países, se contemplaban aspectos técnicos tales como la EIRP máxima, la restricción de la DEP, atenuaciones para emisiones fuera de banda y el HAAT, que son trascendentales a la de hora de implementar un sistema de TVWS. Es por esto, que a continuación se explican estos conceptos para dar claridad sobre este tema. 


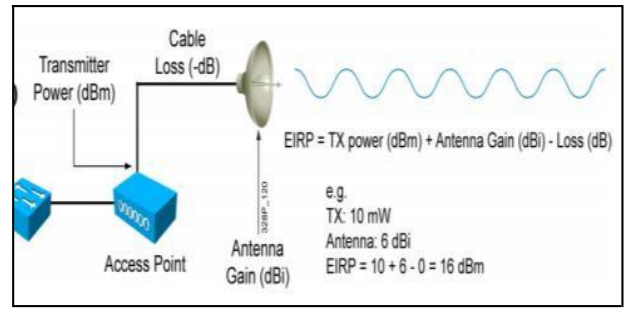

Fig 1. Definición de EIRP. Recuperado de (Henry, 2014)

En la Fig 1 se muestra el EIRP que es la potencia radiada isotrópica efectiva, que consiste: "En la suma de la potencia de transmisión en $\mathrm{dBm}$, con la ganancia de la antena en $\mathrm{dBi}$ menos las pérdidas en el cable en dB". (Henry, 2014)

Asimismo, otro concepto importante es la DEP (Densidad espectral de potencia) como se observa en la Fig 2, que se puede definir como "la potencia media en el ancho de banda de referencia. Aunque el espectro de una señal tenga la misma potencia puede tener diferentes anchos de banda" (ANE, 2017)

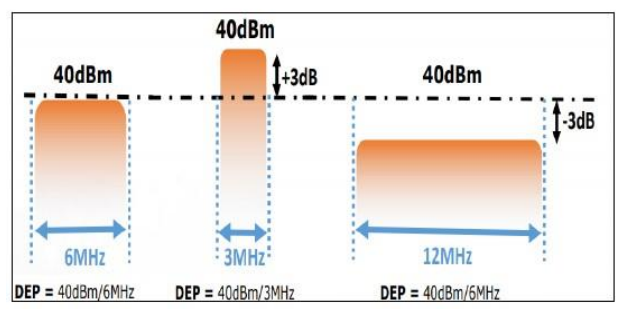

Fig 2. Ejemplos de DEP. Recuperado de (ANE, 2017)

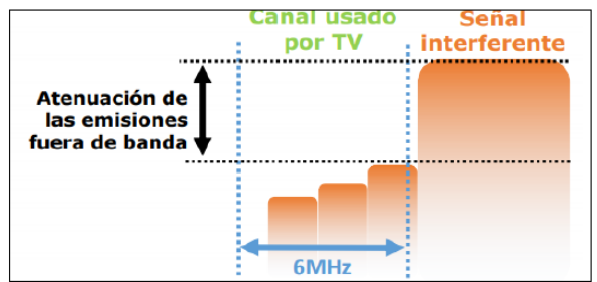

Fig 3. Atenuaciones de las emisiones fuera de banda. Recuperado de (ANE, 2017)

Del mismo modo, se puede explicar que las atenuaciones de las emisiones fuera de banda, que se observan en la Fig. 3 "son emisiones fuera del ancho de banda necesario de la señal". (ANE,
2017) Además, se puede definir el HAAT (altura sobre el promedio del terreno) que se calcula como se observa en la Fig 4.

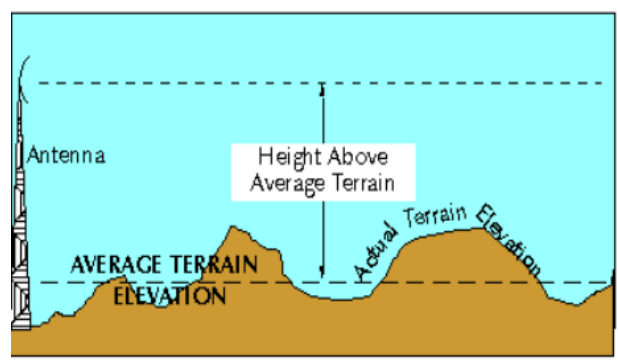

Fig 4. HAAT. Recuperado de (Phillips \& Mylet, 2014)

A partir, de la aclaración de los conceptos anteriormente expuestos se puede llevar a cabo un análisis de la Tabla 2, que posee información acerca de los aspectos técnicos que se manifiestan en la regulación. Así, se logró especificar que algunos países como Australia, Kenia y Malawi aún no han finalizado su regulación, pero ya establecieron algunos aspectos importantes para el uso de los espacios en blanco de TV.

La EIRP máxima, difiere entre los diversos países especialmente en Nueva Zelanda donde se da en unidades $\mathrm{dBw}$, sin embargo, la tendencia entre todos los países es de $36 \mathrm{dBm}$. Con respecto, a la restricción de la DEP, se aclaró que la potencia promedio en el ancho de banda para diferentes países de acuerdo a la regulación puede usar anchos de banda de $8 \mathrm{MHz}$ si es afín al Reino Unido o 6 $\mathrm{MHz}$ si está de acuerdo a la regulación de Estados Unidos.

Las atenuaciones para emisiones fuera de banda cambian en cada país, principalmente en función del tipo de dispositivo, ya que la regulación en los países contemplaba dispositivos fijos, portables, entre otros; todas las potencias de atenuación se dan en unidades de dB. En cuanto, al parámetro HAAT se logró determinar que es similar para todos los países con un valor de $250 \mathrm{~m}$, excepto para el Reino Unido que manifestó que no importa la altura donde se ubiquen las antenas. Además, en Colombia, aunque los parámetros técnicos se ajustaban a la FCC, el HAAT es de $800 \mathrm{~m}$ de acuerdo a lo establecido por la ANE. 
Tabla 2: Aspectos técnicos de la regulación

\begin{tabular}{|c|c|c|c|c|}
\hline País & $\begin{array}{c}\text { EIRP } \\
\text { Máxima }\end{array}$ & $\begin{array}{l}\text { Restricción de } \\
\text { la DEP }\end{array}$ & $\begin{array}{c}\text { Atenuaciones } \\
\text { para emisiones } \\
\text { fuera de banda }\end{array}$ & $\begin{array}{c}\text { HAAT } \\
\text { Máximo }\end{array}$ \\
\hline Alemania & $40 \mathrm{dBm}$ & $40 \mathrm{dBm} / 8 \mathrm{MHz}$ & $-84 \mathrm{dBm} / 100 \mathrm{KHz}$ & $250 \mathrm{~m}$ \\
\hline Australia & $36 \mathrm{dBm}$ & $30 \mathrm{dBm} / 8 \mathrm{MHz}$ & $\begin{array}{c}\text { No está } \\
\text { disponible }\end{array}$ & $250 \mathrm{~m}$ \\
\hline Canadá & $36 \mathrm{dBm}$ & $30 \mathrm{dBm} / 6 \mathrm{MHz}$ & $\begin{array}{c}>- \\
42,8 \mathrm{dBm} / 100 \mathrm{KHz} \\
>55,4 \mathrm{~dB} / 6 \mathrm{MHz}\end{array}$ & $250 \mathrm{~m}$ \\
\hline Colombia & $40 \mathrm{dBm}$ & $40 \mathrm{dBm} / 6 \mathrm{MHz}$ & $>55,4 \mathrm{~dB} / 6 \mathrm{MHz}$ & $800 \mathrm{~m}$ \\
\hline $\begin{array}{c}\text { Estados } \\
\text { Unidos }\end{array}$ & $\begin{array}{l}36 \mathrm{dBm} \\
40 \mathrm{dBm} \\
\text { con } \\
\text { suficiente } \\
\text { espectro }\end{array}$ & $\begin{array}{l}30 \mathrm{dBm} / 6 \mathrm{MHz} \\
40 \mathrm{dBm} / 6 \mathrm{MHz}\end{array}$ & $\begin{array}{c}>- \\
42,8 \mathrm{dBm} / 100 \mathrm{KHz} \\
>55,4 \mathrm{~dB} / 6 \mathrm{MHz}\end{array}$ & $250 \mathrm{~m}$ \\
\hline Finlandia & $20 \mathrm{dBm}$ & $20 \mathrm{dBm} / 8 \mathrm{MHz}$ & $-84 \mathrm{dBm} / 100 \mathrm{KHz}$ & $250 \mathrm{~m}$ \\
\hline Japón & $36 \mathrm{dBm}$ & $30 \mathrm{dBm} / 6 \mathrm{MHz}$ & $\begin{array}{c}12,6 \\
\mathrm{dBm} / 100 \mathrm{KHz} \\
- \\
42,8 \mathrm{dBm} / 100 \mathrm{KHz}\end{array}$ & $250 \mathrm{~m}$ \\
\hline Kenia & $36 \mathrm{dBm}$ & $30 \mathrm{dBm} / 8 \mathrm{MHz}$ & $\begin{array}{c}\text { No está } \\
\text { disponible }\end{array}$ & $250 \mathrm{~m}$ \\
\hline Malawi & $40 \mathrm{dBm}$ & $40 \mathrm{dBm} / 8 \mathrm{MHz}$ & $\begin{array}{c}\text { No está } \\
\text { disponible }\end{array}$ & $250 \mathrm{~m}$ \\
\hline $\begin{array}{c}\text { Nueva } \\
\text { Zelanda }\end{array}$ & $10 \mathrm{dBw}$ & $21 \mathrm{~dB} / 8 \mathrm{MHz}$ & $\begin{array}{c}-36,8 \\
\mathrm{dBm} / 100 \mathrm{KHz} \\
- \\
56,8 \mathrm{dBm} / 100 \mathrm{KHz} \\
-52,8 / 100 \mathrm{KHz}\end{array}$ & $250 \mathrm{~m}$ \\
\hline $\begin{array}{l}\text { Reino } \\
\text { Unido }\end{array}$ & $36 \mathrm{dBm}$ & $30 \mathrm{dBm} / 8 \mathrm{MHz}$ & $\begin{array}{c}\text { Dispositivo } \\
\text { Clase } 5>43 \mathrm{~dB} \\
\text { Clase } 4>54 \mathrm{~dB} \\
\text { Clase } 3>64 \mathrm{~dB} \\
\text { Clase } 2 \text { y } 1>74 \mathrm{~dB}\end{array}$ & $\begin{array}{c}\text { No } \\
\text { importa la } \\
\text { altura }\end{array}$ \\
\hline Singapur & $36 \mathrm{dBm}$ & $30 \mathrm{dBm} / 8 \mathrm{MHz}$ & $17 \mathrm{dBm} / 100 \mathrm{KHz}$ & $250 \mathrm{~m}$ \\
\hline Sudáfrica & $36 \mathrm{dBm}$ & $\begin{array}{c}41,2 \\
\mathrm{dBm} / 8 \mathrm{MHz} \\
36 \mathrm{dBm} / 8 \mathrm{MHz} \\
20 \mathrm{dBm} / 8 \mathrm{MHz}\end{array}$ & $\begin{array}{c}22,2 \mathrm{dBm} / 100 \mathrm{KHz} \\
17 \mathrm{dBm} / 100 \mathrm{KHz} \\
1 \mathrm{dBm} / 100 \mathrm{KHz}\end{array}$ & $250 \mathrm{~m}$ \\
\hline Vietnam & $36 \mathrm{dBm}$ & $30 \mathrm{dBm} / 8 \mathrm{MHz}$ & $17 \mathrm{dBm} / 100 \mathrm{KHz}$ & $250 \mathrm{~m}$ \\
\hline
\end{tabular}

Nota. Datos tomados de las siguientes referencias: (ANE, 2017; CEPT, 2015; Dudda et al., 2012; Ниu, 2015; ICASA, 2018; iDA, 2015; Ishizu, 2015; Mikeka, 2018; NICT, 2015, 2016; RSM, 2018)

\subsection{Análisis de pruebas piloto e implementaciones}

Las pruebas pilotos e implementaciones, que se han realizado respecto a áreas comerciales hablaban de temas como comunicación inalámbrica entre vehículos, redes celulares, implementación de software para optimización de sistemas, pruebas en tiempo real, internet de las cosas, radio cognitiva, entre otras investigaciones con el fin de avanzar en la tecnología TVWS, que prometía ventajas en la implementación de internet en las zonas rurales principalmente.

Asimismo, los espacios en blanco de televisión se han usado con fines sociales, realizando pruebas piloto en lugares apartados; con el fin de brindar internet en escuelas, a campesinos, entre otros, aumentando la cobertura de este servicio tan necesario en la actualidad.

\section{DISCUSIÓN}

Se cumple con el objetivo, de realizar un estudio de la tecnología TVWS en Colombia, ejecutando una comparación con diversos países del mundo en cuanto a regulación, aspectos técnicos, proyectos pilotos e implementaciones. Así se estipula que en el mundo se han realizado diversos estudios en el área de TVWS, debido a las ventajas que presenta en relación con la tecnología WIFI, tales como su alcance, velocidad de transmisión, atravesar obstáculos y llegar a lugares de difícil acceso, principalmente en zonas rurales.

Como futuras investigaciones se propone: Un estudio acerca de los dispositivos de espacios en blanco (WSD), disponibles para realizar implementaciones. También, pruebas de funcionamiento de la tecnología TVWS, en áreas rurales donde el Ejército Nacional ejerce soberanía. Además, pruebas piloto de la tecnología TVWS, en empresas de la región y sus ventajas respecto a otras tecnologías.

\section{CONCLUSIONES}

- Las múltiples pruebas piloto e implementaciones en el mundo, establecen el interés continuo de los países en usar este tipo de tecnologías tanto en fines sociales como en áreas comerciales.

- La regulación en cada país, garantiza que no se cause interferencia a los usuarios primarios y que exista una estandarización de los sistemas TVWS, de acuerdo al país donde se implementen.

- Se determina que Colombia, se destaca por sus avances en regulación TVWS y ha evolucionado significativamente en el desarrollo de este tipo de tecnología tanto en pruebas piloto como en implementaciones.

- Al observar las características de la tecnología TVWS es viable llevar internet a las áreas de 
operaciones del Ejército Nacional mediante el aprovechamiento de los espacios en blanco de TV, en el espectro electromagnético de Colombia.

\section{RECONOCIMIENTOS}

A nuestra institución el Ejército Nacional, que nos brindó la posibilidad de prepararnos como profesionales. A nuestro director de proyecto, que nos apoyó en el desarrollo de este estudio. Además de todas las personas, que colaboraron de alguna manera con la elaboración de este artículo.

\section{REFERENCIAS}

Abeywardana, R. C., Sowerby, K. W., \& Berber, S. M. (2018). Empowering Infotainment Applications: A Multi-Channel Service Management Framework for Cognitive Radio Enabled Vehicular Ad Hoc Networks. En 2018 IEEE 87th Vehicular Technology Conference (VTC Spring) (pp. 1-5). IEEE.

ACMA. (2016). Five-year spectrum outlook The ACMA' s spectrum management work program, (October).

Altintas, O., Watanabe, T., Kremo, H., Tanaka, H., Nakao, H., Tsukamoto, K., \& Tsuru, M. (2016). Design and Experimental Evaluation of a Database-Assisted V2V

Communications System Over TV White Space. Journal of Signal Processing Systems, 83(1), 45-55.

ANE. (2017). TVWS : Normatividad y proyectos. Recuperado de https://www.ane.gov.co/images/ArchivosDes cargables/Comunicaciones/Evento_UMilitar 2017/TVWS_Avanzada.pdf

ANE. (2018). Mesetas Meta conectado por Espacios en Blanco de Televisión (TVWS). Recuperado de https://www.ane.gov.co/index.php/informaci on-de-interes/noticias/754-mesetas-metaconectado-por-espacios-en-blanco-detelevision-tvws

CA. (2014). Rural Broadband Trials Laikipia County Kenya For the Communications Authority of Kenya.

CEPT. (2015). ECC Report 236, (May).

Cerda, H. (1991). Medios, instrumentos, técnicas y métodos en la recolección de datos e información. H. Cerda (Compilador), Los elementos de la investigación, 235-339.

Cho, B., Koufos, K., Ruttik, K., \& Jäntti, R. (2012). Power allocation in the TV white space under constraint on secondary system
Revista Colombiana de Tecnologías de Avanzada

self-interference. Journal of Electrical and Computer Engineering, 2012, 24.

Cuevas-Riz, J. L. (2017). Espacios blancos de la TV para zonas rurales, 1 . Recuperado de https://www.researchgate.net/publication/318 279621_Espacios_blancos_de_la_TV_para_z onas_rurales

Delicado, P. (2016). Medidas para la construcción de un REM ( Radio Environment Map ) de TVWS en la mitad sur del Área Metropolitana de Barcelona, 31. Recuperado de

https://upcommons.upc.edu/bitstream/handle/ 2099.1/22555/Memoria PFC - Pedro

Delicado

Alcantara.pdf? sequence $=4 \&$ is Allowed $=\mathrm{y}$

Dudda, T., Irnich, T., \& Karlsson, J. (2012). How much TV white space is there in Germany? 17th ITG-Fachtagung: Technologies and Andwendungen.

FCC. (2015). Federal Communications Commission, 1-187.

Gopal, S., Kaul, S. K., \& Roy, S. (2018). Optimizing city-wide White-Fi networks in TV white spaces. IEEE Transactions on Cognitive Communications and Networking, 4(4), 749-763.

Haji, M. A. (2014). THE USE OF WHITE SPACES BY Licensing of TV White Space Networks in Licensing of TV White Space Networks in Way forward, (January), 1-11.

Harrison, K., Muthukumar, V., \& Sahai, A. (2015). Whitespace evaluation software (WEST) and its applications to whitespace in Canada and Australia. En 2015 IEEE International Symposium on Dynamic Spectrum Access Networks, DySPAN 2015 (pp. 47-58). https://doi.org/10.1109/DySPAN.2015.73438 49

Hassan, D., \& Ariful, M. D. (2019). Design and Development of a Testbed Prototype for Cognitive Radio Transmission over TV White Space.

Henderson, A. (2014). El arte de elaborar el estado del arte en una investigación Serie técnica de manuales prácticos para el investigador. Recuperado de https://repositoriotec.tec.ac.cr/bitstream/hand le/2238/9145/El estado del arte 2017.pdf? sequence $=1 \&$ is Allowed $=y$

Henry, J. (2014). Troubleshooting Range : Understanding the Cell Size How Large is My Cell ?

Hernández, R. (2014). Metodología de la Investigación. 6ta Edición MrGraw-Hill. Interamericana Editores. Recuperado de 
http://observatorio.epacartagena.gov.co/wpcontent/uploads/2017/08/metodologia-de-lainvestigacion-sexta-edicion.compressed.pdf

Holland, O., Ping, S., Aijaz, A., Chareau, J. M., Chawdhry, P., Gao, Y., ... Kokkinen, H. (2015). To white space or not to White Space: That is the trial within the Ofcom TV White Spaces pilot. IEEE International Symposium on Dynamic Spectrum Access Networks, 11-22. https://doi.org/10.1109/DySPAN.2015.73438 46

Huu, N. (2015). Results of TV White Space trial in case of Vietnam.

ICASA. (2018). Independent communications authority of South Africa, 32(41512), 1-16.

iDA. (2015). Telecommunications Standards Advisory Committee ( TSAC ) Technical Specification Television White Space Devices.

Industry Canada. (2015). White Space Database Specifications, (1).

Ishizu, K. (2015). Standardizations and trials to utilize TV White Space Contents Concept of TV White Space and standardizations Prototype systems Trials.

Masonta, M., \& Mfupe, L. (2013). Field measurements done on operational TVWS trial network in Tygerberg.

Mikeka, C. (2018). Malawi's TV White Space Regulations: A Review and Comparison with FCC and Ofcom Regulations. IEEE Wireless Communications and Networking Conference (WCNC), 1-6.

Mikeka, C., Thodi, M., Mlatho, J. S. P., Pinifolo, J., Kondwani, D., Momba, L., ... Moret, A. (2014). Malawi Television White Spaces (TVWS) Pilot Network Performance Analysis. Journal of Wireless Networking and Communications, 4(1), 26-32. https://doi.org/10.5923/j.jwnc.20140401.04

NICT. (2015). Research and development on TV White Space in NICT.

NICT. (2016). TVWS Overview and R \& D Status at NICT.

Ofcom. (2015). Decision to make the Wireless Telegraphy (White Space Devices) (Exemption) Regulations 2015, 8(December), 1-8. Recuperado de http://www.legislation.gov.uk/uksi/2015/206 6/pdfs/uksi_20152066_en.pdf

Oh, S. W., Ma, Y., Peh, E., \& Tao, M.-H. (2016). $T V$ white space: The first step towards better utilization of frequency spectrum. John Wiley $\&$ Sons.

Orellana, D., \& Sanchez, M. (2006). Técnicas De
Revista Colombiana de Tecnologías de Avanzada

Recolección De Datos En Entornos Virtuales. Revista de Investigación Educativa, 24(1), 205-222. Recuperado de https://www.redalyc.org/pdf/2833/28332188 6011.pdf

Phillips, A., \& Mylet, D. (2014). What is different and what is the same with TVWS technology.

Reidl, L. (2014). Educational research design : current concepts. Investigación en Educación Médica, 1(1), 35-39. Recuperado de http://www.redalyc.org/pdf/292/2922833600 5.pdf

Roncancio, G. (2017). Sistema cognitivo de petición de canales disponibles en la banda de frecuencia de $470 \mathrm{MHz}$ a $698 \mathrm{MHz}$ para el despliegue de IOT.

RSM. (2018). Spectrum Licence Certification Rules for Crown Management Rights ( PIB 39 ). Radio Spectrum Management, (7).

Song, C., Rahman, M. A., \& Harada, H. (2012). TV White Space Technology Trial for The NICT TV Band Device Prototype (ソフトウェア無線). 電子情報通信学会技術研究報告. $S R$, ソフトウェア無線: IEICE technical report, 111(452), 1-8.

Suarez, M. (2018). Tecnología espacios en blanco. Oportunidad de uso eficiente de espectro para incrementar la conectividad en Colombia. Recuperado de https://www.ane.gov.co/images/comunicacio nes2016/presentacion.pdf 